\title{
Challenges of Digital Twin System
}

\author{
C. Monsone ${ }^{1}$, J. Jósvai ${ }^{2}$ \\ ${ }^{1}$ Széchenyi István University, Doctoral School of Multidisciplinary \\ Engineering Sciences \\ Egyetem tér 1, 9026, Gyốr, Hungary \\ E-mail: monsone.cristina@sze.hu \\ ${ }^{2}$ Széchenyi István University, Department of Vehicle Manufacturing \\ Egyetem tér 1, 9026, Györ, Hungary \\ E-mail: josvai@ga.sze.hu
}

\begin{abstract}
Today's manufacturing and assembly systems have to be flexible to adapt quickly to an increasing number and variety of products. The Industry 4.0 conceptualization has several potentials, i.e. flexibility in business and manufacturing processes, where the intelligent and interconnected systems, in particular the Cyber-Physical Production System (CPPS), play a vital role in the whole lifecycle of eco-designed products. In particular, the CPPS represents a suitable way for manufacturers that want to involve their customers, delivering instructions to machines about their specific orders and follow its progress along the production line, in an inversion of normal manufacturing. The development of Info Communication Technologies (ICT) and Manufacturing Science and Technology (MST) enables the innovation of Cyber-Physical Production Systems. However, there are still important challenges that need to be addressed in particular at technological and data analysis level with the implementation of Deep Learning analysis.
\end{abstract}

Keywords: Digital Twin, CPPS, Data, AI, Industry 4.0, IoT, Cloud 


\section{Introduction}

Industry is one of the pillars of the European economy : the manufacturing sector in the European Union accounts for 2 million enterprises, 33 million jobs and 60\% of productivity growth (Eurostat,2018). We stand on the brink of a new industrial revolution, driven by new-generation information technologies such as the Internet of Things (IoT), cloud computing, big data and data analytics, robotics.

They open new horizons for industry to become more efficient, to improve processes and to develop innovative products and services. Recent studies estimate that digitalisation of products and services can add more than EUR 110 billion of annual revenue to the European economy in the next five years. In particular, with the growing deployments of IoT systems, the importance of the concept of a digital avatar - Digital Twin - of a physical thing has gathered significant interest in the recent years. These digital proxies are expected to be built from the domain knowledge of subject matter experts as well as the real time data collected from the devices [1].

The concept of Industry 4.0, or the Fourth Industrial Revolution, has the potential for radically increased system re-configurability and flexibility. At its core, the notion of Cyber-Physical Production System, as the new generation of embedded systems with advanced artificial intelligence and improved communication capabilities, is seen as the key enabling concept that will render production activities more sustainable. This is due to the modular and self-contained nature of cyber-physically formulated equipment and systems that, instead of relying in statically defined and bespoke interconnections, operate in a more open way by considering dynamically establishing and on-demand interactions between the system components [2] .

The intelligence and adaptiveness attributed to this new class of embedded systems therefore not restricted to the computational capabilities of local controllers/devices and can, as well, harness computationally rich cloud environments. The pursue of new industrial automation concepts and solutions, despite being recently fuelled by the latter initiatives, and related developments, is also backed up by more than 20 year of multidisciplinary research. In particular, digitalisation is related to the Internet of Things (IoT) concept and the convergent development of many other technologies discussed in the Fourth Industrial Revolution ( Industry 4.0). As a founding concept, IoT sees all the devices, with embedded sensors, electronics and capabilities to connect to others, as "things". Such things allow to collect and exchange data through internet and, in general, through networks of devices, named as smart objects [3] . 
Big Data analytics, that access the data and promise to provide fast decision-making with the use of smart analytics tools, complement the IoT with further features useful for decision-making support. In such a technology landscape, an important concept is often remarked: the Digital Twin (DT), meant as a system's digital counterpart along its lifecycle. The DT can be considered as a virtual entity, relying on the sensed and transmitted data of the IoT infrastructure as well as on the capability to elaborate data by means of Big Data technologies, with the purpose to allow optimizations and decision-making. In this scope, the DT is often overlapped with advanced simulation. Overall, the DT, as a virtual entity, can regard everything of the physical world, thus physical asset can also be directly taken as a target scope of DT modeling ( Fig.1)[4].

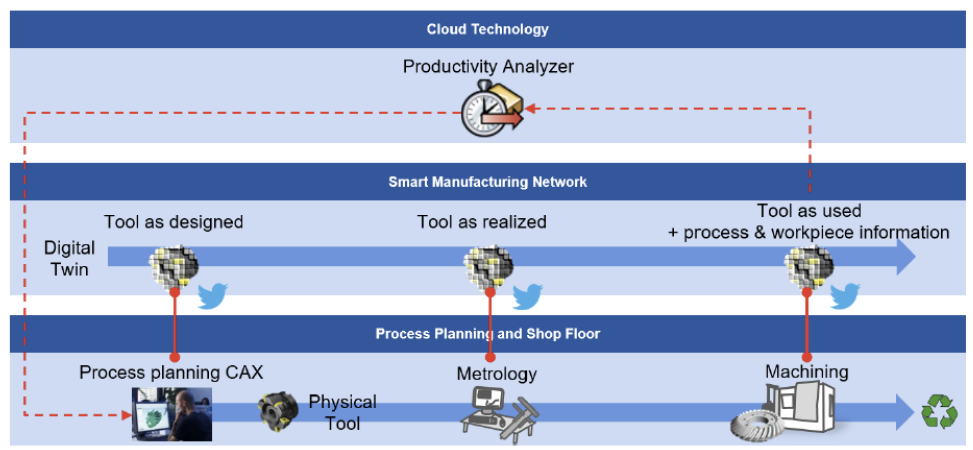

Figure 1. Demonstrator: Digital twin in a production line [5].

\section{State of the Art}

The CPPS conceptualization dramatically reduces the integration effort by virtually eliminating the need, time, and cost for re-programming. The intelligence and adaptiveness attributed to this new class of embedded systems therefore not restricted to the computational capabilities of local controllers/devices and can, as well, harness computationally rich cloud environments, in a multidisciplinary research domain. These eventually led to several innovative production paradigms and technical contributions namely, i.e. Holonic Manufacturing Systems (HMS) powered by Multiagent 
Systems (MAS) and other Artificial Intelligence (AI) techniques. The marriage of digital and physical technologies would affect how customers, consumers, employees, and other parts of the business landscape expect to experience and interact with an organization [4].

Digital twin (DT) is one of the most promising enabling technologies for realizing smart manufacturing and Industry 4.0. DTs are characterized by the seamless integration between the cyber and physical spaces. The importance of DTs is increasingly recognized by both academia and industry. It has been almost 15 years since the concept of the DT was initially proposed. To date, many DT applications have been successfully implemented in different industries, including product design, production, prognostics and health management, and some other fields [5].

Today's manufacturing and assembly systems have to be flexible to adapt quickly to an increasing number and variety of products, and changing market volumes. To manage these dynamics, several production concepts (e.g., flexible, reconfigurable, changeable or autonomous manufacturing and assembly systems) were proposed and partly realized in the past years. In particular, intelligent and interconnected systems play a vital role in the whole lifecycle of eco-designed products and especially for manufacturers that want to involve their customers, delivering instructions to machines about their specific orders and follow its progress along the production line, in an inversion of normal manufacturing [6] .

Based on the collaborative technologies and services mentioned above, the shared workspace between humans and robots can be considered an advanced cyber-physical system, as previous mentioned, the DT. It' is supported by the dynamic control algorithms and online monitoring devices and it's core element is the data acquisition and data evaluation [7].

\section{R\&D Challenges}

The concept of Industry 4.0, or the Fourth Industrial Revolution, has the potential for radically increased system reconfigurability and flexibility and it's aimed at boosting Europe's economy by delivering sustainable economic and social benefits from a digital single market [1].

This is due to the modular and self-contained nature of CPPS formulated equipment and systems that, instead of relying in statically defined and bespoke interconnections, 
operate in a more open way by considering dynamically establishing and on demand interactions between the system components. It is important to notice that the CPPS conceptualization requires a knowledge and data intensive environment as most CPPS components will be collectors and processors.

A Digital Twin can have intelligence. For example, an intelligent product can retrieve information about itself and is capable of participating or making decisions about its own future [8].

Summarizing, several characteristics for an intelligent product are: (a) Requires a global unique identification; (b) Is capable of communicating with its environment; (c) Can retrieve and store data about itself; and (d) Is capable of participating in or making decisions relevant to its own destiny [9]. It is not necessary to have all these characteristics for a Digital Twin, since all these are related to intelligent product. However, some of these characteristics are relevant for the management of the Digital Twin. In particular through CPPS, the development of new business models, new services are expected which may change many aspects of our life. The potential application fields are almost endless: air- and ground-traffic; discrete and continuous production systems; logistics; medical science, energy production, infrastructure surrounding us, entertainment, and we could keep on enumerating[6].

In the coming space only some of the $R \& D$ challenges are outlined from the much bigger set of research fields which are related to CPPS. Especially when adopted in manufacturing, the DT has taken a new objective: to simulate the complex behaviour of production systems, also including external factors, as human presence and technical constraints [10]. In particular:

- Data evaluation and acquisition [7].

- Context-adaptive and (at least partially) autonomous systems. Methods for comprehensive, continuous context awareness, for recognition, analysis and interpretation of plans and intentions of objects, systems and participating users, for model creation for application field and domain and for self-awareness in terms of knowledge about own situation, status and options for action are to be developed [6] .

- Cooperative production systems. New theoretical results are to be achieved 
and the development of efficient algorithms for consensus seeking, cooperative learning and distributed detection is required[6].

- Improved maintenance decision making (damage / cracks prediction; material geometric / plastic deformation, and reliability modelling of physical systems) [11].

- Checking the feasibility and optimizing the control software of the system [12].

- Simulating the orchestration of IoT devices [13] .

- Human-machine (including human-robot) symbiosis. The development of a geometric data framework to fusion assembly features and sensor measurements and fast search algorithms to adapt and compensate dynamic changes in the real environment is required [6] .

- Statistically-based decision making and optimization, such as optimizing the system's behaviour / performances, by simulating it during the design phase or during other lifecycle phases, knowing its past and present states [14][15].

\subsection{Cyber Physical Production System}

The concept of a cyber-physical production system (CPPS) is a manufacturingcentered version of a CPS. The CPS includes embedded systems, internet services, management processes. The CPPS fuses computer science (CS), ICT, and manufacturing-science technology. Based on the collaborative technologies and services mentioned above, the shared workspace between humans and robots can be considered an advanced cyber-physical system, which is supported by the dynamic control algorithms and online monitoring devices. In particular, the CPPS will enable and support the communication between humans, machines and products alike (Fig. 2).

The elements of a CPPS are able to acquisition and process data, and can selfcontrol 


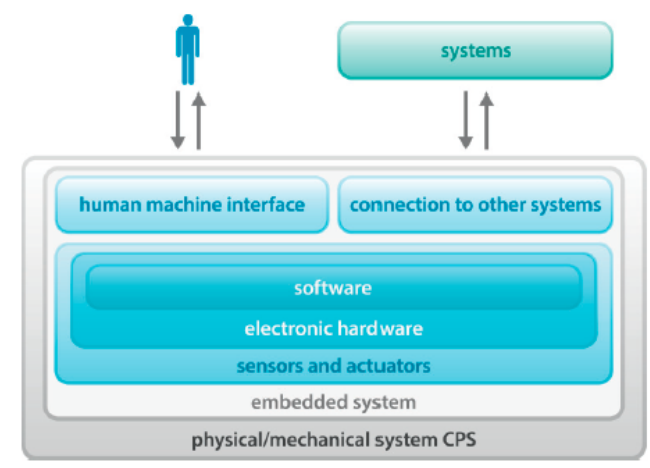

Figure 2. Interaction between Humans and machine in CPS.[6]

certain tasks and interact with humans via interfaces. CPPS partly break with the traditional automation pyramid (left side of Fig. 3).

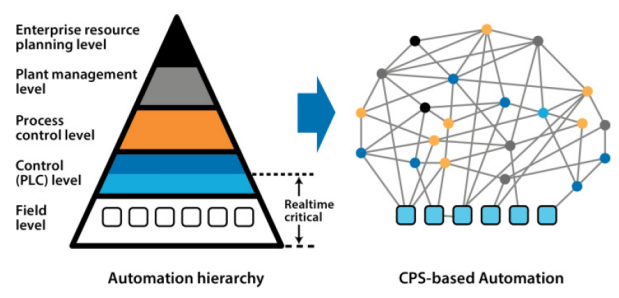

Figure 3. Decomposition of automated hyerarchy with distributed services.[6]

The typical control and field levels still exist which includes common PLCs close to the technical processes to be able to provide the highest performance for critical control loops, while in the other, higher levels of the hierarchy a more decentralized way of functioning is characteristic in CPPS[6].

Currently, the CPPS concept is still under development. In the area of asset management, CPPS has the potential to provide self-awareness and self-maintenance 
capabilities. The implementation of predictive analytics as part of the CPPS framework enables the assets to continuously track their own performance and health status and predict potential failures. By implementing this predictive analytics along with a decision support system, proper services could be requested and actions taken to maximize the uptime, productivity and efficiency of the industrial systems. CPPS, as the central hub for data management in fleet level, plays a critical role in achieving the above-mentioned goals[16].

\subsection{Digital Twin}

Thanks to the CPPS and AI platform, a radically increased system where the re- configurability and flexibility allows a predictive planning and control in order to prevent and solve the potential failure in a production or in a physical system . Indeed, the technological basis of Industry 4.0 roots back in the Internet of Things (IoT), which proposed to embed electronics, software, sensors, and network connectivity into devices (i.e. "things"), in order to allow the collection and exchange of data through the internet[16] [17].

As such, IoT can be exploited at industrial level: devices can be sensed and controlled remotely across network infrastructures, allowing a more direct integration between the physical world and virtual systems, and resulting in higher efficiency, accuracy and economic benefits. Although it is a recent trend, Industry 4.0 has been widely discussed and its key technologies have been identified among which Cyber-Physical Production Systems (CPPS) have been proposed as smart embedded and networked systems within production systems[18] .

They operate at virtual and physical levels interacting with and controlling physical devices, sensing and acting on the real world [19]. According to scientific literature, in order to fully exploit the potentials of CPPS and IoT, proper data models should be employed, such as ontologies which are explicit, semantic and formal conceptualizations of concepts in a domain [20][21][22].

They are the core semantic technology providing intelligence embedded in the smart CPPS and could help the integration and sharing of big amounts of sensed data [23] [24]. Through the use of Big Data analytics, it is possible to access sensed data, through smart analytics tools, for a rapid decision making and improved productivity[25]. The Digital Twin (DT) is meant as the virtual and computerized counterpart of a physical system that can be used to simulate it for various purposes, exploiting a 
real-time synchronization of the sensed data coming from the field; such a synchronization is possible thanks to the enabling technologies of Industry 4.0 and, as such, the DT is deeply linked with it [26].

The DT was first born in the aerospace field - the first definition of the DT was forged by the NASA as "an integrated multi-physics, multi-scale, probabilistic simulation of a vehicle or system that uses the best available physical models, sensor updates, fleet history, etc., to mirror the life of its flying twin. It is ultra-realistic and may consider one or more important and interdependent vehicle systems": this definition first appeared in the draft and after in the final release of the NASA " Modeling, Simulation, Information Technology Processing Roadmap" in 2010 and only recently has been adopted also in manufacturing contexts: such a term is used in industrial environments and in governmental research initiatives. However, scientific literature that describes the contextualisation of the concept in the manufacturing domain is still at its infancy [27].

Digital twins are becoming a business imperative, covering the entire lifecycle of an asset or process and forming the foundation for connected products and services and allows analysis of data and monitoring of systems to head off problems before they even occur, prevent downtime, developing, in a cloud-based system, new opportunities and even plan for the future by using simulations, thinking of a digital twin as a bridge between the physical and digital world[28].

However, Industry 4.0 is a multi-faceted problem, and it is unlikely that all aspects of it will be applicable to all businesses. Whilst the area of Intelligent Manufacturing is itself a multifaceted problem, the recurring element that underpins much of this revolution is the collection, utilization and understanding of data, or the study of 'Informatics'; almost all of the areas linked with the intelligent manufacturing research area rely on the capture and analysis of data in some way.

To this end the use of advanced data analytics and machine learning is a key technology to develop to further these other technologies; and the next step in this chain lies in utilizing the vast reserves of data through data mining and knowledge discovery, to better understand these manufacturing processes[28][29]. 


\section{Methodologies}

Cyber Physical Production Systems (CPPS) have been proposed as a key concept of Industry 4.0 architectures. A CPPS can be described as a set of physical devices, objects and equipments that interact with a virtual cyberspace through a communication network. Each physical device will have its cyber part as a digital representation of the real device, culminating in the "Digital Twin". So, the Digital Twin can monitor and control the physical entity, while the physical entity can send data to update its virtual model [28] [30].

\subsection{Data acquisition}

The Digital Twin model can be composed by different kind of models and data, but for its the realization the following two main systems are introduced for data acquisition: sensor based tracking and machine vision. In particular, the new advanced methodologies considers AutomationML and Deep Convolutional Neural Network (DCNN)[27] [28] [30].

\section{- AutomationML}

The AutomationML is considered one of the best solution for the DT because it stores engineering information following the object-oriented paradigm and allows the modelling of physical and logical plant components as data objects en-capsulating different aspects . An object may consist of other sub-objects, and may itself be a part of a larger composition or aggregation [19]. The AutomationML defines Computer Aided Engineering Exchange (CAEX) as a meta model for the storage and exchange of engineering models. The topics below, summarizes its parts and the way it is used to create models [28].

- Creates a hierarchy of components, called Instance- Hierarchy (IH), from the top-level down to single components (InternalElements, IEs) with interfaces (External Interfaces, EIs) and relations (Internal Links, ILs),

- Reusable System Unit Classes (SUCs) defining component types.

- Reusable Interface Classes (ICs) for specifying connection points of RCs ( Role Classes), SUCs and the interface type of EIs, 
- Attributes for describing characteristics of each previously introduced modeling element. By definition, CAEX supports object-oriented modeling for all of these aspects. External Interfaces describing System Unit Classes instances are connected by Internal Links via External Interfaces, which in turn are instances of Interface Classes [29].

Another way is represented by the utilisation of a Deep Learning techniques, in particular Deep Convolutional Neural Network (DCNN), for the data aquisition , especially where also the aspect of the product is a crucial element in the production line.

- Deep Convolutional Neural Network (DCNN)

In the field of the Deep Learning (DL) the pre-trained Deep Convolutional Neural Network (DCNN) model with transfer learning have shown to be highly effective in processing visual data, such as images and videos. DCNNs take raw input data at the lowest level and transforms them by processing them through a sequence of basic computational units to obtain representations that have intrinsic values for classification in the higher layers [30].

The use of models allows that a user, without knowledge about programming, to model a Digital Twin of the equipment that he operates and create models to exchange data between systems.

\section{Conclusion}

The marriage of digital and physical technologies would affect how customers, consumers, employees, and other parts of the business landscape expect to experience and interact with an organization. While the technologies associated with Industry 4.0 - from robotics to the Internet- of-Things, and from big data analytics to artificial intelligence - are transforming business processes, an often-overlooked challenge is managing the inevitable shift in workplace dynamics, which is crucial to supporting 
the successful integration of Industry 4.0 technologies. As presented in this paper, we have demonstrated that the : one of the key requirements is realizing a real-world production environment that comprises the characteristics of a cyber- physical production system in relation to the data availability and data analysis through AI techniques . Thanks to the Digital Twin, the aim is to allow production models to be implemented using captured near-real time data to improve the production monitoring and to be designed to include the possibility to flexibly configure all of the control-relevant methods and parameters in the production environment. Within this context, it was shown that in terms of attaining production objectives and increasing an enterprise's competitiveness, there is generally tremendous potential both in compiling, supplying and analyzing operational data. As reported in the previuos paragraph, the CPPS and AI platform - through Digital Twin - represents a radically increased system where the re-configurability and flexibility allows a predicitive planning and control in order to prevent and solve the potential failure in a production or in a physical system. In particular, it emerged that the relevance of Digital Twin ( DT) for manufacturing industry lies in their definition as virtual counterparts of physical devices. These are digital representations based on semantic data models that allow running simulations in different disciplines, that support not only a prognostic assessment at design stage (static perspective), but also a continuous update of the virtual representation of the object by a real time synchronization with sensed data. This allows the representation to reflect the current status of the system and to perform real-time optimizations, decision making and predictive maintenance according to the sensed conditions.

\section{References}

[1] Digitisation Research and Innovation - Transforming European industry and services, August 2017.

https://publications.europa.eu/en/publication-detail/

- /publication/662617ce-d4b9-11e7-a5b9-01aa75ed71a1/

language-en

[2] K. Ashton, That 'internet of things' thing, RFID journal, 22 (7) (2009) pp. 97-114.

[3] L. Atzori, A. Iera, G. Morabito, The internet of things: A survey, Computer networks, 54 (15) (2010) pp. 2787-2805. 
doi:10.1016/j.comnet.2010.05.010

[4] F. Tao, H. Zhang, A. Liu, A. Y. Nee, Digital twin in industry: state-of-the-art, IEEE Transactions on Industrial Informatics, 15 (4) (2018) pp. 2405-2415. doi:10.1109/TII.2018.2873186

[5] D. Botkina, M. Hedlind, B. Olsson, J. Henser, T. Lundholm, Digital twin of a cutting tool, Procedia CIRP, 72 (2018) pp. 215-218. doi:10.1016/j.procir.2018.03.178

[6] L. Monostori, Cyber-physical production systems: Roots, expectations and RD challenges, Procedia Cirp, 17 (2014) pp. 9-13.

doi:10.1016/j.procir.2014.03.115

[7] T. H. J. Uhlemann, C. Lehmann, R. Steinhilper, The digital twin: Realizing the cyber-physical production system for industry 4.0, Procedia Cirp, 61 (2017) pp. 335-340.

doi:10.1016/j.procir.2016.11.152

[8] B. Dworschak, H. Zaiser, Competences for cyber-physical systems in manufacturing-first findings and scenarios, Procedia CIRP, 25 (2014) pp. 345-350. doi:10.1016/j.procir.2014.10.048

[9] K. Hribernik T. Wuest, K. D. Thoben, Towards Product Avatars Representing Middle-of-Life Information for Improving Design, Development and Manufacturing Processes, in: G. L. Kovács, D. Kochan (eds) Digital Product and Process Development Systems. NEW PROLAMAT 2013. IFIP Advances in Information and Communication Technology, vol 411. Springer, Berlin, Heidelberg, 2013, pp. 85-96.

doi:10.1007/978-3-642-41329-2_10

[10] R. Rosen, G. Von Wichert, G. Lo, K. D. Bettenhausen, About the importance of autonomy and digital twins for the future of manufacturing, IFAC-PapersOnLine, 48 (3) (2015) pp. 567-572.

doi:10.1016/j.ifacol.2015.06.141

[11] A. Cerrone, J. Hochhalter, G. Heber, A. Ingraffea, On the effects of modeling asmanufactured geometry: toward digital twin, International Journal of Aerospace 
Engineering, 2014 (Article ID 439278) (2014) pp. 1-10. doi:10.1155/2014/439278

[12] M. Schluse, J. Rossmann, From simulation to experimentable digital twins: Simulation-based development and operation of complex technical systems, in 2016 IEEE International Symposium on Systems Engineering (ISSE) (2016) pp. $1-6$.

doi:10.1109/SysEng.2016.7753162

[13] A. Canedo, Industrial IoT lifecycle via digital twins, in Proceedings of the Eleventh IEEE/ACM/IFIP International Conference on Hardware/Software Codesign and System Synthesis (2016) pp. 1-29.

[14] T. Gabor, L. Belzner, M. Kiermeier, M. T. Beck, A. Neitz, A simulation-based architecture for smart cyber-physical systems, in 2016 IEEE International Conference on Autonomic Computing (ICAC) (2016) pp. 374-379.

doi:10.1109/ICAC.2016.29

[15] J. Ríos, F. M. Morate, M. Oliva, J. C. Hernández, Framework to support the aircraft digital counterpart concept with an industrial design view, International Journal of Agile Systems and Management, 9 (3) (2016) pp. 212-231.

doi:10.1504/IJASM.2016.079934

[16] J. Lee, H. D. Ardakani, S. Yang, B. Bagheri, Industrial big data analytics and cyber-physical systems for future maintenance service innovation, Procedia Cirp, 38 (2015) pp. 3-7.

doi:10.1016/j.procir.2015.08.026

[17] J. Lee, B. Bagheri, H. A, Kao, A cyber-physical systems architecture for industry 4.0-based manufacturing systems, Manufacturing letters, 3 (2015) pp. 18-23. doi:10.1016/j.mfglet.2014.12.001

[18] C. Legat, C. Seitz, S. Lamparter, S. Feldmann, Semantics to the shop floor: towards ontology modularization and reuse in the automation domain, IFAC Proceedings Volumes, 47(3) (2014) pp. 3444-3449.

doi:10.3182/20140824-6-ZA-1003.02512 
[19] N. Jazdi, Cyber physical systems in the context of Industry 4.0, in: 2014 IEEE international conference on automation, quality and testing, robotics, ClujNapoca, 2014, pp. 1-4.

doi:10.1109/AQTR.2014.6857843

[20] M. Garetti, L. Fumagalli, E. Negri, Role of ontologies for CPS implementation in manufacturing, Management and Production Engineering Review, 6 (4) (2015) pp. 26-32.

doi:10.1515/mper-2015-0033

[21] E. Negri, L. Fumagalli, M. Garetti, L. Tanca, Requirements and languages for the semantic representation of manufacturing systems, Computers in Industry, 81 (2016) pp. 55-66.

doi:10.1016/j.compind.2015.10.009

[22] T. R. Gruber, Toward principles for the design of ontologies used for knowledge sharing?, International journal of human-computer studies, 43 (5-6) (1995) pp. 907-928.

doi:10.1006/ijhc.1995.1081

[23] S. Borgo, An ontological approach for reliable data integration in the industrial domain, Computers in Industry, 65 (9) (2014) pp. 1242-1252.

doi:10.1016/j.compind.2013.12.010

[24] S. Heymans et al. Ontology Reasoning with Large Data Repositories, in: M. Hepp, P. De Leenheer, A. De Moor, Y. Sure (eds) Ontology Management. Computing for Human Experience, vol 7. Springer, Boston, 2008, pp. 89-128.

doi:10.1007/978-0-387-69900-4_4

[25] J. Davis, T. F. Edgar, J. Porter, J. Bernaden, M. Sarli, Smart manufacturing, manufacturing intelligence and demand-dynamic performance, Computers Chemical Engineering, 47 (2012) pp. 145-156.

doi:10.1016/j.compchemeng.2012.06.037

[26] J. Lee, H. A. Kao, S. Yang, Service innovation and smart analytics for industry 4.0 and big data environment, Procedia Cirp, 16 (2014) pp. 3-8.

doi:10.1016/j.procir.2014.02.001 
[27] E. Negri, L. Fumagalli, M. Macchi, A review of the roles of digital twin in cps-based production systems, Procedia Manufacturing, 11 (2017) pp. 939-948. doi:10.1016/j.promfg.2017.07.198

[28] Representation of process control engineering requests in PI Diagrams and for data exchange between PID tools and PCE-CAE tools, IEC PAS 62424:2006 (2006).

[29] G. N. Schroeder, C. Steinmetz, C. E. Pereira, D. B. Espindola, Digital twin data modeling with automationml and a communication methodology for data exchange, IFAC-PapersOnLine, 49 (30) (2016) pp. 12-17.

doi:10.1016/j.ifacol.2016.11.115

[30] K. Gopalakrishnan, S. K. Khaitan, A. Choudhary, A. Agrawal, Deep Convolutional Neural Networks with transfer learning for computer vision-based data-driven pavement distress detection, Construction and Building Materials, 157 (2017) pp. 322-330.

doi:10.1016/j.conbuildmat.2017.09.110 